\title{
DISTRIBUTED DATABASES - SOME APPROACHES, MODELS AND CURRENT TRENDS
}

\section{S. Klisarova-Belcheva}

Faculty of Economic and Social Sciences, Plovdiv University "Paisii Hilendarski”, Plovdiv, Bulgaria

\begin{abstract}
The progress of internet technology in recent years increases requirements toward distributed platforms to store huge amounts of data. The purpose of this article is to present an overview of the most common currently used distributed databases (like NoSQL and NewSQL). The patterns of storage of the latter, their advantages and disadvantages in different architectural solutions and development trends are discussed. In conclusion, the paper emphasizes the increased importance of distributed platforms for business software.
\end{abstract}

Key words: distributed databases, big data, NoSQL, NewSQL

\begin{abstract}
DISTRIBUTED DATABASES - SOME APPROACHES, MODELS AND CURRENT TRENDS

The revolution of the Internet technology in recent years has increased the necessity of distributed platforms for storage of huge volumes of data. This paper aims to give an overview of the most famous distributed databases (like NoSQL and NewSQL). It compares the patterns of storage, their advantages and disadvantages in different architectural solutions for storage and development trends.
\end{abstract}

\section{INTRODUCTION}

The progress of the technologies for data storage and information processing set the database (DB) as core of the modern software applications. Database concept appeared in the 60 s of the XX century in the context of automated information management. Distributed database management system (DDBMS) are created in response to the necessity of the scalable database for developing web applications. Web-based and scalable database spread rapidly in recent years, becoming a popular name NoSQL DB.

The aim of this report is to juxtapose newer models for data storage and traditional relational model comparing their basic characteristics by applying a set of classifications. The paper emphasis are the possibilities and applications of distributed databases (NoSQL) and distributed relational databases NewSQL.

\section{DISTRIBUTED DATABASES - GENERAL CHARACTERISTIC AND CLASSIFICATIONS}

In the 70 s of the last century, working on the theory of data storing, Edgar F. Codd creates a relational data model. Nowadays this model is in the basis of the leading database management systems (DBMS). The relational database management systems (RDBMS) are an integral part of the implementation of modern business applications. The main characteristic that gives the name of the model is a relation between objects in the database. Foundation of the relational model is the support for ACID transactions. ACID is an acronym used to describe the basic properties of transactions in the RDB: Atomicity, Consistency, Isolation and Durability. Distributed DB are scalable. Their main feature is the possibility of physical separation of data among multiple networked computers.

The second important feature of the DDBMS is the absence of relations, i.e. NoSQL database offer free scheme of stored information. The free scheme increases the possibility of storing large volumes of information in a damage resistant architecture. Rick Cattell identified the main disadvantage of the DDBMS compared to the transactional 
model, which is that they do not support ACID transactions (1).

In 2000, prof. Eric Brewer formulated a conjecture (now known as Brewer's Theorem), stating that any form of distributed computing is capable of providing at the same time not more than two of the three aforementioned properties: consistency of the data, availability and partition tolerance (distributed). Two years later Seth Gilbert and Nancy Lynch managed to formally prove Brewer's assumption (2). Brewer's Theorem is the basis for construction of the first from the reviewed DB classifiers distributed and undistributed database. Undistributed DB provide both properties consistency and availability, as most cases these databases are relational. In 2013 A. Nayak explores the types of DDBMS depending on which properties provide availability and/or consistency (3).

Another possible database classification is represented by Cattell, here DBMS are classified according to the storage place of the information - the database stored in RAM (in memory) and DB stored on external memory (HDD) (1). The implementation of modern database stored on external drive, have developed special file systems for example, HDFS (Hadoop Distributed File System) - file system FOR special purposes, GFS (Google File System) - distributed parallel file system WITH protection from breaks, Amazon S3 network file system and others.

In a study of NoSQL databases Jing Han offers a classification based on the physical model for information storage: row-oriented stores, column-oriented stores (wide column too), document stores, key-value stores and graph stores (4).

Cattell added also a classification depending on the mechanisms for accessing and modifying the data. Relational and some of the distributed DBMS provide ACID transactions, while others are using MVCC (Multi Version Concurrency Control), locks (provide a mechanism to allow only one user at time to read or modify record) and others.

There are specific mechanisms developed to access the data in some distributed systems. One of the most popular models for distributed computing is MapReduce. Hung Yang analyzes the application of MapReduce to extract aggregate information as a prerequisite for the development of scalable parallel applications for processing large volumes of data distributed on multiple networked computers (5). Grigoriev and
Cviashchenko explore the main types of mechanisms for replication and data consistency (6). Depending on the type of replication (synchronous and asynchronous) the different systems have variety of mechanisms for consistency, like two phase commit and etc.

\section{DISTRIBUTED RELATIONAL DBMS - CURRENT TRENDS AND BASE COMPARISON}

\begin{tabular}{|c|c|l|l|}
\hline 2015 & 2014 & Data base & Type \\
\hline 1 & 1 & Oracle & Relational \\
\hline 2 & 2 & MySQL & Relational \\
\hline 3 & 3 & MS SQL Server & Relational \\
\hline 4 & 51 & MongoDB & Document \\
\hline 5 & $4 \downarrow$ & PostgreSQL & Relational \\
\hline 6 & 6 & DB2 & Relational \\
\hline 7 & 7 & MS Access & Relational \\
\hline 8 & $9 \uparrow$ & Cassandra & Wide-column \\
\hline 9 & $8 \downarrow$ & SQLite & Relational \\
\hline 10 & $13 \uparrow$ & Redis & Key-value \\
\hline
\end{tabular}

Figure 1. DB rating by type and years

According to a recent research on the prevalence of databases, there is a steady trend of increasing interest in NoSQL DBMS shown on Figure 1 (7).

Despite the increased interest in DDBMS, leading places in the rankings still hold traditional RDBMS - Oracle, MySQL и MS SQL Server. The results show that the relational model is still predominant, but there is a steady trend of the increasing use of distributed systems. It occurs a new alternative to relational and distributed database management system which is up to the needs combining the capabilities of both the traditional and the NoSQL database. These new database systems, known as NewSQL, actually are NoSQL which supports the control of transactions and SQL (like Join, union) etc.

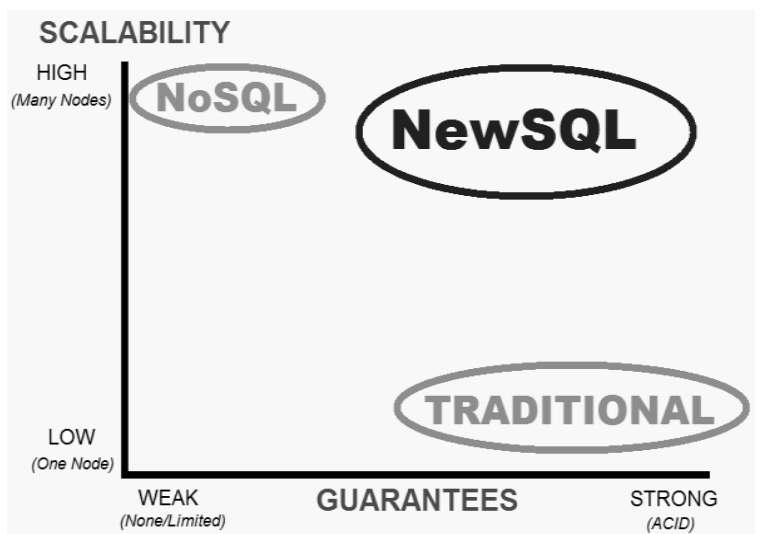

Figure 2. Comparison between traditional, NoSQL and NewSQL database

Thus, the distributed and the relational databases have opportunities, to integrate the 
advantages of both traditional relational and the distributed database. Figure 2 shows NewSQL database management systems as an union of the strong features of relational and distributed database - ACID transactions and scalability.

\begin{tabular}{|r|c|c|c|}
\hline PROPERTIES & $\begin{array}{c}\text { TRADITION } \\
\text { AL SOL }\end{array}$ & NOSOL & NEWSOL \\
\hline ACID PROPERTY & & $\varkappa$ & \\
\hline IN MEMORY DB & $\varkappa$ & & \\
\hline BIG DATA & $\varkappa$ & & \\
\hline RDBMS & & $\varkappa$ & \\
\hline
\end{tabular}

Figure 3. Basic properties of traditional RDBMS, NoSQL and NewSQL

Figure 3 summarizes the properties of the three main types of database, such as ACID transactions, support large data, relations and the possibility of storage in memory. The figure shows that while NoSQL and Relational DBMS are complementary, the NewSQL provides all of their properties.

The main representatives of NewSQL are:

- NuoDB - scale-out SQL database for cloud computing and the modern datacenters

- VoltDB - In-memory performance, streaming analytics with millisecond latency, OLTP in a scale-out architecture, SQL and JSON with ACID guarantees, Hadoop ecosystem integration

- Clustrix - roves that SQL can scale out in production to massive deployment sizes

- SAP Hana - an in-memory computing platform that has completely transformed RDB industry

- FoundationDB - stores data in the KeyValue Store.

- Google Spanner - Spanner is Google's distributed NewSQL DB, the successor to BigTable etc.

NewSQL is an established trend with a number of options, which fits relational and distributed database model.

\section{DESCRIPTION AND COMPARISON OF MODERN DBMS BY MODEL OF DATA STORAGE}

To assess the advantages and disadvantages of each model, it is necessary to compare their abilities of realization of basic operations for data processing: inserting, updating, deleting, select (select or aggregates) and metadata updating (add or modification of the attributes).

\section{a. Row-oriented DBMS}

Systems using row-oriented model of the record, are appropriate in the treatment of multiple transactions, related mainly to the addition of rows and requests. Data is stored sequentially in areas called rows (records). Each record in the database is represented by a fixed length which is relatively constant. When there is a need to add a new line, it is added at the back, without the need of complex operations. In John Kubiatovicz's comparative analyses to add a new attribute and to change or remove it is a quite complex operation, because the values of each row are written sequentially according to the attributes in a table (9). The principle scheme of the manner to preserve data shown in Figure 4. The major representatives of row-oriented database are most used relational databases: Oracle database, Microsoft SQL Server, MySQL, DB2 (IBM), PostgreSQL. These databases support OLTP (online transaction processing) and are used in business software such as software for managing sales in supermarkets, ATM, etc.

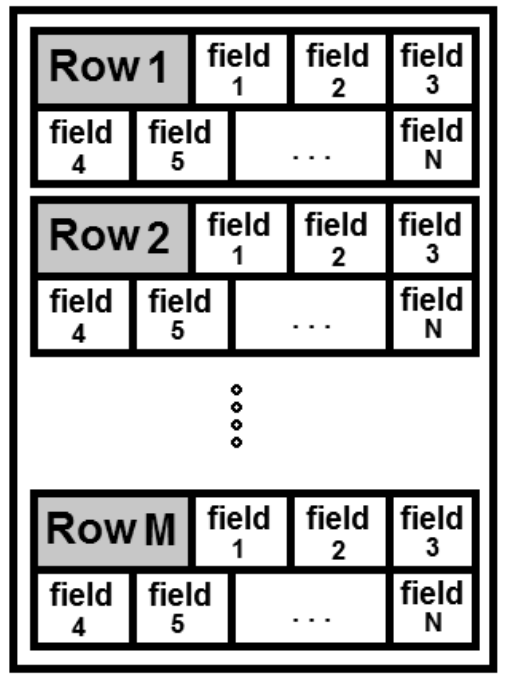

Figure 4. Presentation of data in row-oriented database

Row-oriented database are appropriate in the following situations: short transactions covering a small number of records, frequent updates, many users and fast response time.

\section{b. Column-oriented database}

In the column-oriented model the information is stored in areas that correspond to the attributes of the table. Operations for adding and extracting an information from rows (multiple attributes) are much more complex than adding, editing or extracting information from certain columns. The advantage is in case to edit a column that all data is from the same type and the editing could be done without affecting other rows. Kubiatowicz, analyzing the fast working of these databases compared 
to row-oriented, experimentally proves that a column-oriented databases are more effective in calculating the aggregate functions (9). The scheme of the model represented on the Figure 5.

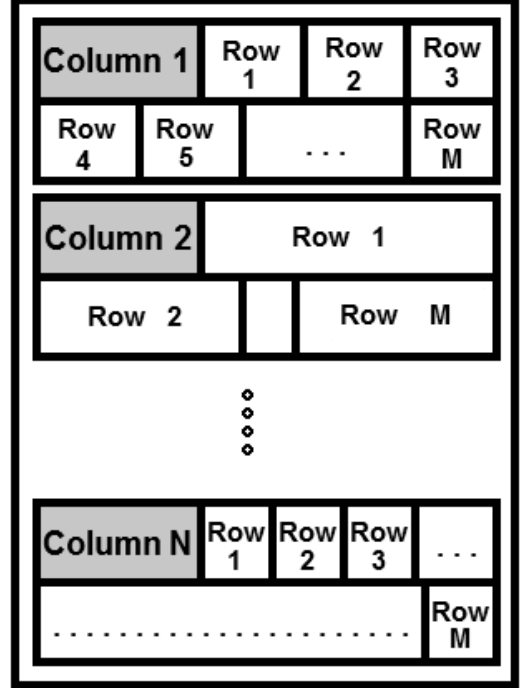

Figure 5. Presentation of data in column-oriented database

Key representatives of the column-oriented databases are: Cassandra (Apache), HyperTable - Open Source system which works with Apache HDFS, BigTable - Google, HBase - Apache Hadoop, Accumulo etc.

\section{c. Key-value stores}

This is the simplest model of data storage. Its fields of data can be regarded as "identical." Each field contains two main attributes:

- key that is synthetic or can be automatically generated

- value - most often String / BLOB storage of various types of data in a common format like JSON, XML, etc.

Presentation of data is based on the similarity of the areas in which they are stored. All operations are trivial, and because of the specificity of the model, the operations for change of attributes (metadata) are missing.

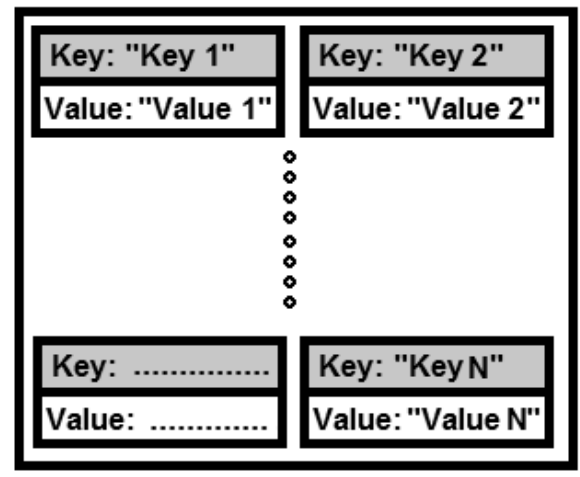

Figure 6. Presentation of data in the key-value stores

DB type key-value use associative arrays, known as a dictionary or a card (map). All operations for creating, editing, deleting or extraction are facilitated.

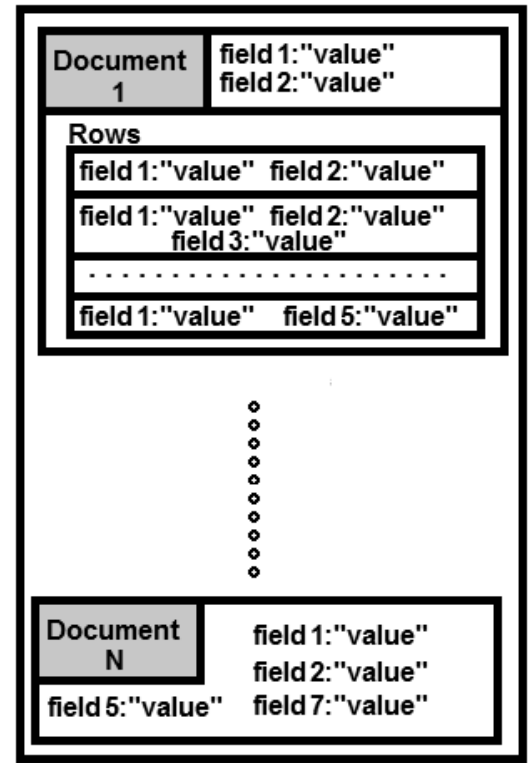

Figure 7. Presentation of data in the documentoriented stores

This approach to implementation is applied in relational database in order to reduce the size of the base. The model is known as the EAV (Entity-Attribute-Value) data model. In a study of Carlo Beltrame about the specifics of the model key-value is demonstrated that this model is the preferred solution for the creation of small non-commercial applications due to the simplified structure and reduced size of the database [10]. Among the most popular databases of this class are: Dynamo - by Amazon, Voldemort - used in LinkedIn, Redis, Scalaris - unlike most other NoSQL $\mathrm{DB}$, it ensures strict consistency, ACID transactions etc.

\section{d. Document-oriented DB}

In document-oriented models using the technology known from key-value databases and the data (values) are presented in a more complex structure called document. As a rule are used formats such as ...XML, JSON, YAML and BSON. The main advantage is that the data include the description of the structure (metadata). Due to the use of descriptive language these computer sets are also known as XML-oriented database. Various implementations include different data organization such as collections, tags and more. Databases of this type resemble a relational logical representation of data. The main operations to remove data for each document are fast. Extracting parts of all documents, however, is time-consuming and complex task. The possibility of calculating the aggregates is achieved by secondary indexes and realization of MapReduce algorithm for processing the data. 
The main representatives of this class database are: CouchDB - Apache's development of web applications, data is stored in JSON format, MongoDB - by many features, it is similar to CouchDB, the difference is in the replication an automatic sharding and MapReduce on aggregate operations on documents.

\section{e. Graph-oriented stores}

Among the more rarely used models are NoSQL graph-oriented and hierarchical databases. Their main feature is the relations between the elements. The elements are simple structures called nodes. Relations are made with a table of links between nodes. They are defined as features for each relation. These databases are suitable for solving tasks for the realization of complex interrelationships. Presentation in graph helps to accelerate speedup for processing data.

Key representatives of the graph-oriented database are:

- Neo4j-ACID transactions, scales to billions of nodes. This is the most popular graph DB.

- $\quad$ ArangoDB - document and graph database

- InfoGrid - Web Graph database

The main operations are quick insertion, but operations data retrieval can be time consuming.

\section{f. Comparison of the different models}

Row-oriented databases are essentially relational databases. The main advantages of this model are in simultaneous processing of multiple transactions and inquiries. The system has to react immediately to customer inquiries (OLTP - Online transaction processing). The advantages of the column-oriented DBMS are easily calculable aggregates. They are used mainly for data warehouses, CRM and other ad hoc inquiry systems, where aggregate are computed over large data items - OLAP (Online analytical processing). An example is the use of column-oriented database in banking software solutions. Example software solutions include RDBMS and column-oriented store. Interface for export/import data between the different databases. This leads to easier use aggregates and successful analyses.

By examining trends in business analysis of large data Karthik Kambatlaa shows that when the database is larger than the RAM memory, or the aggregates are more important than the flow of data, a column-oriented database significantly improves the recovery of data for analysis [8]. Column-oriented databases are suitable in the case of adding new attributes. This essential is the design of the database.

Document-oriented databases are suitable in case of need for multiple data into one document, which expresses the current status of the document (snapshot). They are suitable for blogs or documentation systems that require multiple data regarding each document, such as a state at the time of entering information (snapshot). For them it is important retrieving the information about the document to be implemented at once, not just parts of it. These systems can be successfully used for archiving documents created in relational databases. Most systems for DB management of the documentaryoriented model, offer fast text search. Many of them also offer secondary indexes, regardless the structure of the information. Another advantage is that things do not have the pattern of the base used is pre-clarified. In a columnoriented database structure is easily changeable, but it is required initially to set a structure that eventually changes according to adding new attributes.

Disadvantages of document-oriented database in comparison to relational are:

- Does not support document structure both advantage and disadvantage

- It is often slower than relational

- $\quad$ Requires more storage space

- Does not support of protection from repeated information.

- Possibility for "broken document"

Because of the possibility of random structure for each node of document-oriented database, there is possibility the document to not be fully described. Document-oriented database is improved model of key-value model. They provide an opportunity to describe the indexes of documents and internal attributes, and their deficiency results in the requirement for clarification of the scheme.

\section{PRACTICAL EXAMPLES OF THE DIFFERENT DB}

Giants in the Internet industry, generating substantial portion of Web traffic rely increasingly on NoSQL database. Kai Ored classifies No SQL DB [11] and use in the Twitter, Facebook, LinkedIn, eBay, Amazon. For example Cassandra is used in eBay. It is mainly used because of the large nomenclatures - 200+ million items, 100 million. users, 2 billion pages are exhibited per day. Casandra is used for the following reasons: multi datacenter (active-active), availability - no SPOF, scalability, write performance, distributed counters and hadoop support. Ebay also use MongoDB and HBase. Other large companies that use Casandra because of the mentioned advantages above are: Soundcloud, Spotify, Instagram, Adobe Audience Manager, Adobe digital market 
suite, AOL, British Gas, Call of Duty, Cisco, VMWare, Dell and many other big companies. EBay use MongoDB like McAfee, US Department of energy, US National Archives. The authoritative source for McAfee threat information, MongoDB enables big data analytics and supports the real-time flow of cyberthreat data between Global Threat Intelligence's cloud-based system and end client products. It currently stores 4 billion documents - terabytes of data. The advantages they use are:

- Easy to increase storage capacity by orders of magnitude,

- Lowered latency, easy to interact with JSON

- GridFS for high availability \&CDNf

- Flexability to "Decorate" base data

- Atomic updates and full consistency

They use : OS - Cent OS, Deployment platform - their own, 24 servers with 96 GB RAM per server and SSD, sharding and replication - 6 shards in largest cluster, database -4 billions documents, 20'000 writes per second on SSDs. Google uses its own product BigTable over GFS.

MariaDB is the database that powers billions of users on sites like Google and Wikipedia. MariaDB is using from reuter.de, Ingenico financial solutions, Jetair, Swisscom, Transticket and etc.

Despite the increased data volumes general architect, of the System Engineering Team at Jetair, wanted a system which delivered faster transactions. He felt there were better options available as, "Our previous provider did not give any support for load balancing using Distributed Replicated Block Device (DRBD), and MySQL High Availability (MHA) support for unlimited amount of servers was overpriced."

\section{CONCLUSION}

The Global digital market, automated production and the need to analyze business information in real time require reliable software that supports fast transactions with large volumes of data.The problem of choosing a suitable database (relational, distributed for OLAP or OLTP) will continue to be a general task in the development of software applications. More software architects utilize the possibilities offered by the distributed database in their systems combining them with traditional relational.

From the review of modern DBMS data according to their physical model can be concluded that a major part of web applications and part of business applications stake on the system model containing multiple data management system - traditional and distributed.

Acknowledgements This paper was sponsored as a part of project SR15 FESS 019 / 24.04.2015 at the Scientific Research Fund of the University of Plovdiv "Paisii Hilendarski".

\section{REFERENCES}

1. Rick Cattell, Scalable SQL and NoSQL Data Stores, SIGMOD Record, December 2010

2. Seth Gilbert, Nancy A. Lynch, Perspectives on the CAP Theorem, Computer 45(2): Feb. 2012

3. A Nayak, A Poriya, D Poojary, Type of NoSQL Databases and its Comparison with Relational Databases, International Journal of Applied Information Systems, March 2013

4. Jing Han, Survey on NoSQL database, 6th International Conference on Pervasive Computing and Applications (ICPCA), 2011

5. Hung-Chih Yang, Ruey-Lung Hsiao, D. Stott Parker, Map-Reduce-Merge: Simplified Relational Data Processing Large Clusters, SIGMOD'07, Beijing, China, June 12-14, 2007

6. Grigoriev U. A., Cviashchenko E. V., Analysis processing versions of records in databases NoSQL, Science and Education Publisher VPO MSTU. N.E. Bauman.", FC $77-48211$

7. Aslett, Matthew "How Will The Database Incumbents Respond To NoSQL And NewSQL?", 451 Group, published 2011-0404

8. Karthik Kambatlaa, Giorgos Kolliasb, Vipin Kumarc, Ananth Gramaa, Trends in big data analytics, Journal of Parallel and Distributed Computing 74, Issue 7, July 2014

9. John Kubiatowicz, C-Store / DB Cracking, EECS 262a, Advanced Topics in Computer Systems, 29.10.2014

10.Carlo Beltrame, Key-value stores, Algorithms for Database Systems, ETH Zurich, 2013 April 26

11.Kai Ored, Analysis and Classification of NoSQL Databases and Evaluation of their ability to replace an Object-relational Persistent Layer, Technical University Munich,2010 April 14, 